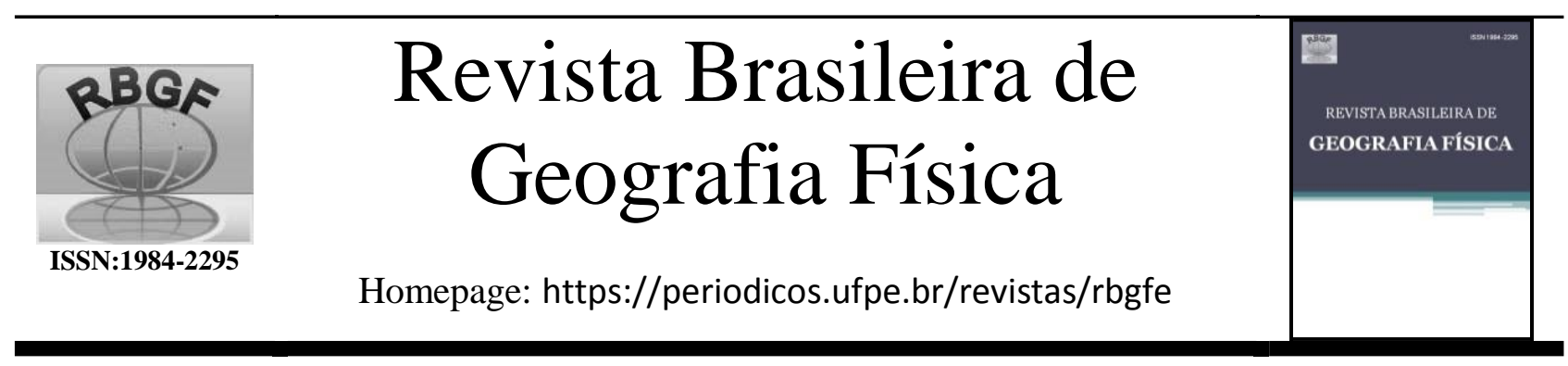

\title{
Uma Análise Visual da Espacialização das Descargas Elétricas Atmosféricas e do Comportamento das Mortes Causadas por Raios no Estado da Bahia
}

\author{
Ubiraci Alves Muniz Barretto ${ }^{1}$, Renato Lima Novais ${ }^{2}$, Frederico Jorge Ribeiro Barbosa ${ }^{3}$
}

\begin{abstract}
${ }^{1}$ Mestrando do Programa de Pós-Graduação em Engenharia de Sistema e Produtos, ubarretto@gmail.com, Instituto Federal de Educação, Ciência e Tecnologia da Bahia (IFBA) - R. Emídio dos Santos, s/n - Barbalho, Salvador/BA. ${ }^{2}$ Dr. em Ciência da Computação, Professor do Departamento de Computação, renato@ifba.edu.br, Instituto Federal de Educação, Ciência e Tecnologia da Bahia (IFBA) - R. Emídio dos Santos, s/n - Barbalho, Salvador - BA. ${ }^{3}$ Doutorando em Ciência da Computação, Profess or do Departamento de Computação, fred.barboza@gmail.com, Instituto Federal de Educação, Ciênciae Tecnologia da Bahia (IFBA) - R. Emídio dos Santos, s/n - Barbalho, Salvador - BA
\end{abstract} Artigo recebido em 15/02/2021 e aceito em 14/06/2021

\section{R E S U M O}

Estudos divulgados pelo Grupo de Eletricidade Atmosférica do Instituto Nacional de Pesquisas Espaciais colocam o Brasil como o país com maior incidência de descargas atmosféricas no mundo sendo, em média, 77,8 milhões todos os anos. No período de 2000 a 2019, foram registrados 2.194 óbitos causados por raios no país, sendo que a cada 50 mortes por raios no mundo, uma é no Brasil. Este trabalho se propõe a realizar uma análise visual do comportamento das descargas atmosféricas no estado da Bahia, relacionando-a a o número de mortes causadas por raios em cada município. O objetivo é investigar se há alguma rela ção entre o número de mortes, a região e a população. Para tal, foram consultados os dados de densidade de descargas atmosféricas e número de mortes causados por raios divulgados pelo INPE e Ministério da Saúde, respectivamente. Também foram utilizados os dados referentes ao número de habitantes disponibilizados pelo IBGE em cada município do estado da Bahia. A análise visual foi realizada com a utilização da ferramenta VYR. Os resultados mostraram uma maior incidência de descargas na região Oeste do estado. Quanto ao número de mortes, verificou-se uma distribuição homogênea no estado, com uma a parente relação com a área e número de habitantes dos municípios. Observou-se uma redução no número de mortes nos últimos dez anos, provavelmente relaciona da com o aumento do grau de instrução da população.

Palavras-chaves: Análise visual, descargas elétricas atmosféricas, mortes causa das por raios e estado da Bahia

\section{A Visual Analysis of the Spatialization of Atmospheric Electric Discharges and Behavior of Deaths by Rays in the State of Bahia}

\begin{abstract}
A B S T R A C T
Studies released by the Atmospheric Electricity Group of the National Institute for Space Research place Brazil as the country with the highest incidence of lightning in the world, with an average of 77.8 million every year. In the period from 2000 to $2019,2,194$ deaths from lightning were recorded in the country, with every 50 deaths from lightning in the world, one is in Brazil. The objective of this work is to carry out a visual analysis of the behavior of lightning strikes in the state of Bahia, relating it to the number of deaths caused by lightning in each municipality. The goal is to investigate whether there is any relationship between the number of deaths, the region and the population.To this end, data on lightning density density and number of deaths caused by lightning were published by INPE and the Ministry of Health, respectively. The number of inhabitants provided by IBGE in each municipality in the state of Bahia was also used. Visual analysis was performed using the VYR tool. The results showed a higher incidence of discharges in the western region of the state. As for the number of deaths, there was a homogeneous distribution in the state, with an apparent relationship with the number of inhabitants of the municipalities. There was a reduction in the number of deaths in the last ten years, probably related to the increase in the education level of the population.
\end{abstract}

Keywords: Visual analysis, lightning strikes, lightning deaths and the state of B ahia 


\section{Introdução}

"Brasil, o país em que mais caem raios no mundo". Comeste título, Guilherme Eler inicia sua reportagem publicada na revista Superinteressante (Eler, 2020). Na reportagem, o autor destaca que o Brasil é recordista mundial de descargas elétricas sendo, em média, 77,8 milhões todos os anos e por volta de 300 atingem pessoas.

Esses números são oriundos do relatório apresentado pelo Grupo de Eletricidade Atmosférica (ELAT) do Instituto Nacional de Pesquisas Espaciais (INPE). Segundo o relatório, 2012 foi o ano com maior incidência de raios, registrando 94,3 milhões. Em 2013 foram 92 milhões, em 2014 foram 62,9 milhões e em 2015, 68,6 milhões de raios. Neste último ano houve um acréscimo devido ao fenômeno El Niño, responsável pelo aumento dos raios nas regiões Sul e parte das regiões Sudeste e Centro-Oeste (Eler, 2020). Um estudo a nível global realizado por Lau (2021), utilizando-se dos dados oriundos da Rede Mundial de Localização de Relâmpagos, identificou, entre outros resultados, um aumento no número de relâmpagos entre os trópicos no período de 2012 - 2015.

Em relação às mortes causadas por raios, um levantamento elaborado pelo ELAT reuniu informações coletadas pelo Departamento de Informações e Análise Epidemiológica (CGIAE) do Ministério da Saúde, veículos da imprensa e dados de população do Instituto Brasileiro de Geografia e Estatística (IBGE), no período de 2000 a 2019. Os dados revelam um total de 2.194 fatalidades registradas: uma média de 110 casos por ano no período. Além disso, mostram que a cada 50 mortes por raios no mundo, uma é no Brasil. Sendo mais de 200 feridos e causando, a cada ano, prejuízos de um bilhão de reais (ELAT, 2020).

Em 2007, um estudo realizado pelo ELAT junto às empresas do setor elétrico, telecomunicações, seguro, equipamentos eletroeletrônicos, construção civil e aviação, já contabilizava valores bilionários em prejuízos anuais. O prejuízo ao setor elétrico é o maior com cerca de 600 milhões de reais, seguido pelas empresas de telecomunicações com cerca de 100 milhões de reais e as empresas seguradoras e de eletroeletrônicos com cerca de 50 milhões de reais cada (INPE, 2007). O prejuízo total é bem menor que o prejuízo que os raios causam nos Estados Unidos, de cerca de 2 bilhões de dólares (aproximadamente 4,4 bilhões de reais), apesar de ocorrer no Brasil cerca do dobro de raios em comparação aos Estados Unidos (INPE, 2007).

No estado da Bahia, reportagem divulgada pelo portal G1, destacou que nas 24 horas do dia 27/10/2020 o estado registrou mais de $100 \mathrm{mil}$ raios. Neste dia, na região Oeste, no município de Luís Eduardo Magalhães, uma fazenda foi incendiada após ser atingida por uma descarga elétrica. Entre as cidades com maior incidência estavam Barreiras e Riachão das Neves (Bahia, 2020).

Segundo o Doutor Osmar Pinto Júnior, Coordenador do ELAT, a Bahia possui um grande contraste. Na região Oeste e Sul se têm a mais alta incidência de raios. Já na região Norte e Leste, incluindo Salvador, a incidência é menor. Ainda segundo Osmar, o período compreendido entre meados de outubro até meados de março no estado é conhecido como temporada dos raios, devido a maior incidência (Bahia, 2020).

O Relatório do INPE (2011), que divulgou o ranking de incidência de raiosno Brasil no biênio 2009-2010, apontou duas observações relevantes: a forte correlação entre a incidência de raios e a urbanização e o aumento de $11 \%$ na incidência de raios desde 2005 até 2010 em municípios commais de 200 mil habitantes. Estas observações foram alvo das pesquisas realizadas por Sousa (2012). No estudo, o autor buscou avaliar com mais detalhes, do ponto de vista estatístico, estas duas observações apontadas pelo relatório do INPE. O estudo se concentrou na região Centro-Sul do Brasil e utilizou uma série histórica para medir a densidade de descargas atmosféricas no período de 2005 - 2010. Através de uma análise estatística, o autor concluiu que de fato há correlação entre a incidência de raios e a urbanização, entretanto não pode afirmar sobre significativo crescimento na densidade de raiosem municípios com mais de 200 mil habitantes.

Conceição (2013), realizou estudo semelhante para o estado de São Paulo. Para o autor, além dos fatores climáticos, as características do relevo e o fator urbanização, aparentemente, também influenciam na diferença das ocorrências de descargas elétricas registradas no estado. Realizou o mapeamento da incidênciade descargas do período de 2005-2006, analisando tanto este fenômeno quanto às vítimas registradas no mesmo período. Concluiu que o período estudado foi muito curto para se fazer qualquer afirmativa. 
Ainda para o estado de São Paulo, Santos (2016a) realizou uma análise da identificação dos eventos climáticos extremos de descargas atmosféricas sobre o mesmo, nas escalas anual e sazonal. Para tal, utilizou os dados da Rede Integrada Nacional de Detecção de Descargas (RINDAT) e da Rede Brasileira de Detecção de Descargas Atmosféricas (BrasilDAT) para o período de 1999 a 2014. De sua pesquisa, revelou que no período de maior atividade elétrica, as descargas tendem a se concentrar no Centro-Leste do estado e no período de menor atividade elétrica, tendem a se concentrar sobre o Sudoeste.

Também utilizando os dados do RINDAT e BrasilDAT, Santos (2016b) analisou as descargas atmosféricas do tipo nuvem-solo durante o verão no estado de São Paulo. Através do método não paramétrico de Mann-Kendall, calculou a tendência da incidência de descargas. Os eventos extremos foram determinados usando a técnica dos Percentis. Seus resultados demonstraram que há uma alta concentração de descargas na região Centro-Leste do estado. Observou, também, que existe uma tendência crescente da incidência de descargas.

Gomes (2019), voltou sua pesquisa para realização de um estudo da incidência de descargas atmosféricas no município de Governador Valadares, estado de Minas Gerais, nos anos de 2012 a 2015. Detalhando os métodos utilizados para definição dos comparativos de densidade de descargas atmosféricas, verificou a possibilidade de relação entre densidade de descargas atmosféricas e altitude. Através da análise dos mapas de densidade de descargas atmosféricas e relevo, concluiu que as regiões com maiores densidades de raios são as que possuem em seu território elevadas altitudes. Sugeriu, portanto, que há uma relação da distribuição de descargas atmosférica com o relevo do município.

Com proposta semelhante, Gin (2012) apresentou um estudo com a atividade mensal e a distribuição espacial das descargas elétricas atmosféricas nos estados de São Paulo e Paraná durante o período de outubro de 1998 a fevereiro de 1999. No trabalho, realizou um comparativo dos índices obtidos nestes estados com os de outros países.

Heilmann (2018), utilizou os dados do RINDAT, para descargas atmosféricas, num período de 19 anos para o litoral do estado do Paraná. Considerando valores de pico de corrente média das descargas atmosféricas, realizou uma análise da distribuição espacial dos raios e sua relação com a topografia. Concluiu que cidades com maior altitude possuem maior probabilidade de incidência de raios.

Utilizando os dados oriundos do projeto GoAmazon e a seleção de atributos pelo método do algoritmo de árvore de decisão, Albrecht (2020) apresentou uma análise da distribuição espacial das descargas elétricas na região Central da Bacia Amazônica. Os seus resultados demonstraram que com a referida técnica foi possível identificar os padrões associados ao aumento do número de descargas elétricas, assim como na classificação de nuvens com e sem raios.

Algumas pesquisas também se voltaram a identificar a relação da incidência de raios com outros fenômenos físicos. O trabalho de Ribeiro (2018), por exemplo, utilizou os dados de sondagem da atmosfera, feitas diariamente no aeroporto de Belém, com objetivo de criar um modelo estatístico, baseado no métodode árvore de decisão, com intuito de encontrar uma relação entre os índices termodinâmicos da atmosfera e a ocorrência de raios. Já Pompeu (2018), utilizando os dados de raios coletados pela rede STARNET, realizou uma análise do comportamento de tempestades severas visando acompanhar os sistemas atmosféricos produtores de raios e sua relação com a precipitação. Também utilizando os dados da STARNET, Sodré (2020) investigou a relação da quantidade de raios do tipo nuvem-solo em função da altitude e do relevo.

Em outros países pesquisas desta natureza foram realizadas. Porras (2018), estudou a densidade de descargas do tipo nuvem-solo na Colômbia num período de quatorze anos. Já Lyu (2018), investigou as características climáticas da atividade de relâmpagos na região Noroeste do pacífico. Seltzer (2020), apresentou a primeira climatologia de uma década com relâmpagos para Europa e, por fim, Tian (2021) examinou as variações de relâmpagos do Sul da Ásia com base no mais longo conjunto de dados de relâmpagos existentes das observações OTD/LIS.

Voltando-se para as análises das mortes por raios, Bortolin (2019) realizou um estudo das ocorrências de descargas atmosféricas na região de maior ocorrência do fenômeno dentro da Bacia Hidrográfica do Rio Paraíba do Sul (São Paulo), analisando as formas e implicações dessas ocorrências na sociedade com base nos números e nas circunstâncias dos óbitos. Utilizou a base de dados do ELAT no período de 1998 a 2011. Ao final, entendeu que a principal causa do elevado número de óbitos por descargas atmosféricas no país é reflexo da falta de conscientização da população comrelação às formas de se protegerdas mesmas. 
Já Leal (2019), realizou uma análise estatística de mortes e acidentes por raios na região da Amazônia Legal, no período de 2009 a 2019, com intuito de verificar a extensão dos danos causados por este fenômeno à população local. Relacionou o número de mortes e acidentes com a ocorrência de raios observando que cerca de $38 \%$ dos atingidos por raios na região viam a óbito.

No contexto internacional, foram identificadas pesquisas que analisaram as mortes causadas por raios e suas possíveis consequências. Antonescu (2018), analisou as mortes causadas por raios na Romênia no período entre 1999 e 2015. Identificou uma redução de aproximadamente 35,7 $\%$ no número de mortes quando comparado o período de 1999 a 2003 com 2011 a 2015. Argumentou que uma redução maior pode ser obtida através de campanhas informativas e atividades de mitigação. Pesquisas semelhantes foram realizadas por Fan (2021) na China, Cooper (2018) na Malásia, Banwari (2021) e Holle (2019) na África e Yadava (2020) na Índia. Segundo Ferreira (2018), as lesões causadas por raios são a segunda maior causa de mortes relacionadas com o clima nos Estados Unidos, cerca de 300 feridos e 100 mortes estão associadas anualmente com relâmpagos.

Considera-se pertinente a realização deste estudo, visto nãoterem sidoidentificados trabalhos semelhantes realizados para o estado da Bahia. Diante deste fato, o trabalho tem como objetivo realizar uma análise visual do comportamento da densidade de descargas atmosféricas e das mortes causadas por raios no estado. Nossa intenção é investigar se a hipótese de que há relação do número de raios com região específica e população é verdadeira para o estado da Bahia.

Especificamente, busca-se avaliar as características de distribuição da incidência de descargas no estado e a existência de relação entre esta distribuição e o número de mortes causadas por raio nos municípios. Ao utilizar a análise visual, buscou-se otimizar o estudo dos dados, pois ela facilita as observações, verificação do comportamento e relação entre as grandezas.

Os dados referentes aos valores de densidade de descargas atmosféricas foram obtidos através de relatórios e consulta ao portal do ELAT, compreendendo o período de 2000 a 2019. Através de consulta ao DATASUS (Ministério da Saúde), foram obtidos os valores referentes ao número de mortes causadas por raios também no período compreendido entre os anos 2000 e 2019. Por fim, foram obtidos os dados referentes ao número de habitantes dos municípios baianos do ano de 2019, provenientes de consulta ao IBGE. Após processo de extração e tratamento dos dados, estes foram inseridos na ferramenta de análise visual VYR (Visualize Your Region) (Deiro e Novais, 2021) onde foram obtidas as imagens para análise.

Ao final, observou-se uma maior incidência de descargas na região Oeste e Centro Sul do estado. Foi verificada uma redução no número de óbitos causadas por raios no período estudado, mas não se identificou possível relação entre a incidência de descargas atmosféricas e o número de óbitos causados por raios nos municípios. Concluiu-se que a quantidade de fatalidades deve estar relacionada com o grau de instrução da população e densidade demográfica do município.

Além desta Introdução, a estrutura deste trabalho é organizada como se segue. Na Seção 2 são definidos os procedimentos metodológicos utilizados e na Seção 3 são apresentados os resultados obtidos e as discussões relacionadas a análise dos dados. Para finalizar, na Seção 4, é apresentada a conclusão.

\section{Material e métodos}

Os dados de incidência de raios para o estado da Bahia, foram obtidos através das informações divulgadas pelo ELAT/INPE em dois recortes. O primeiro, trata-se de relatório divulgado pelo INPE (2013) onde é apresentada uma média de densidade de descargas ( $\left.\mathrm{km}^{2} / \mathrm{ano}\right)$ do período entre 1998 e 2011, para todos os municípios do Brasil. Deste relatório, foram extraídos os dados referentes aos municípios do estado da Bahia. O segundo recorte trata-se de acesso ao portal do ELAT (2020) onde, através de consulta individual por município, é possível obter a média da densidade de descargas atmosféric as para o período compreendido entre os anos de 2016 a 2019. Através do comparativo dos números destes dois intervalos, foi possível construir uma distribuição da densidade de descargas dos municípios baianos nas últimas duas décadas. Não foram localizados os dados de densidade referentes ao período de 2012 a 2015 para os municípios do estado da Bahia.

Quanto aos dados relacionados ao número de mortes por raios no estado, consulta realizada à base de dados do DATASUS (DATASUS, 2020) do Ministério da Saúde, permitiu obter o quantitativo de mortes a cada ano, distribuídos por município, no período entre os anos de 2000 a 2019.

Ainda para o estudo, foram obtidos os números de habitantes estimados para cada município no ano de 2019 disponibilizados pelo IBGE. 
Para realização do comparativo da relação de densidade de descargas atmosféricas, númerode mortes e quantidade de habitantes entre os municípios, foi utilizado o coeficiente de letalidade semelhante ao apresentado por Conceição (2013).

O coeficiente de letalidade seria o risco de morte na população exposta a um evento de descargas elétricas atmosféricas (Conceição 2013, Apud Laurenti et al., 1987) e será avaliado com relação a densidade de descargas atmosféricas, sendo obtida pela relação:

$n^{\mathrm{o}}$ de mortes causadas por raios

$\overline{\text { densidade de descar gas atmosf éricas no município }}$

E pela relação com o número de habitantes do município dado por:

$\frac{n^{\circ} \text { de mortes causadas por raios } * 1000}{n^{\circ} \text { total de habitantes do município }}$

Para obtenção dos valores do coeficiente de letalidade com relação a densidade de descargas atmosféricas nos municípios no período de 2000 a 2019, foi calculada a média de densidade de descargas entre os dois valores obtidos para os recortes de 1998 a 2011 e 2016 a 2019.

Segundo Tufte (2011), uma forma efetiva de encontrar informações importantes em grandes massas de dados com várias dimensões é visualizando figuras que correspondem a estes números, ou seja, aplicando técnicas de visualização. Desta forma, os dados foram tratados, compilados e importados para a ferramenta de análise visual de dados VYR com o intuito de se realizar as análises dos mesmos.

Neste caso, a técnica de visualização utilizada será a análise de mapas. Os mapas são componentes de visualização universalmente conhecidos, e que podem ser usados como background para visualizações que tenham informações geográficas, frequentemente na forma de coordenadas pontuais ou de regiões geográficas (Grégio, 2009).

A análise foi realizada comparando-se os mapas do estado da Bahia para as informações referentes a densidade de descargas atmosféricas e número de mortes causadas por raios com intuito de verificar o comportamento e possível relação entre estes fenômenos.

$\mathrm{Na}$ Tabela 01 é apresentada a estatística descritiva dos dados utilizados na VYR.

Tabela 1 - Estatística descritiva dos dados.

\begin{tabular}{clcccc}
\cline { 3 - 5 } Ano & & \multicolumn{4}{c}{ Estatística Descritiva } \\
\hline \multirow{2}{*}{2011} & Atributos & Mínimo & Máximo & Média & Desvio Padrão \\
\hline \multirow{2}{*}{2019} & População & 2612,00 & 2675656,00 & 33613,68 & 136041,91 \\
& Qtd Descargas & 0,21 & 9,05 & 1,85 & 1,32 \\
& Mortes & 0,00 & 5,00 & 0,14 & 0,48 \\
\hline & População & 3669,00 & 2953986,00 & 36797,24 & 150566,70 \\
& Qtd Descargas & 0,07 & 6,03 & 1,65 & 1,11 \\
& Mortes & 0,00 & 5,00 & 0,14 & 0,48 \\
\hline
\end{tabular}

\section{Resultados e discussão}

\section{Região de estudo}

Localizada na Região Nordeste do Brasil, a Bahia faz limite com oito estados brasileiros, são eles: Espírito Santo, Minas Gerais, Goiás, Tocantins, Piauí, Pernambuco, Sergipe e Alagoas, além de ser banhada pelo oceano Atlântico. Sua extensão territorial é de $564.692,669 \mathrm{~km}^{2}$, sendo o maior estado nordestino e o quinto maior do Brasil. Possui 417 municípios e, conforme contagem populacional realizada em 2010 pelo IBGE, o estado tem 14.016.906 habitantes. A densidade demográfica é de $24,8 \mathrm{hab} / \mathrm{km}^{2}$ e o crescimento demográfico é de $0,7 \%$ ao ano. É a maior população do Nordeste e a quarta maior do país (Cerqueira, 2021).
Três tipos climáticos se observam no estado da Bahia: o clima quente e úmido sem estação seca, o clima quente e úmido com estação seca de inverno e o clima semiárido quente. $\mathrm{O}$ primeiro domina ao longo do litoral, com temperaturas médias anuais de cerca de $23^{\circ} \mathrm{C}$ e totais pluviométricos superiores a $1.500 \mathrm{~mm}$. O segundo caracteriza todo o interior, com exceção da parte setentrional e do vale do São Francisco. Apresenta temperaturas médias anuais que variam entre $18^{\circ} \mathrm{C}$ nas áreas mais elevadas e $22^{\circ} \mathrm{C}$ nas áreas mais baixas, e totais pluviométricos equivalentes a mil milímetros. $\mathrm{O}$ terceiro tipo climático é encontrado no norte do estado e no vale do São Francisco. As temperaturas médias anuais superam $24^{\circ} \mathrm{C}$, mas a pluviosidade é inferior a 700 mm (Alcoforado, 2003). 
Espacialização da densidade de descargas atmosféricas

Os atuais sistemas de detecção de relâmpagos são baseados na medida da radiação eletromagnética gerada pelo fenômeno, por um conjunto de sensores distribuídos no solo em uma adequada configuração. Dependendo da frequência da radiação medida, diferentes aspectos dos relâmpagos podem ser investigados (ELAT, 2020).

Por meio de processamento computacional, que variará de acordo com o método de medição utilizado, o ponto de incidência da descarga é calculado e georreferenciado (Gomes, 2019). Neste caso, é medida a densidade de descargas que incidem por ano em um quilômetro quadrado (Paulino, 2016). Através do resultado destas medições, o portal do ELAT permite consultar os dados da densidade de descargas elétricas $\left(\mathrm{km}^{2} / \mathrm{ano}\right)$ em todos os municípios do País. Desta forma, as informações extraídas para o estado da Bahia, e lançadas na ferramenta VYR, possibilitam visualizar a distribuição da densidade de descargas entre os municípios do estado com as médias obtidas dos intervalos entre 1998-2011 e 2016-2019.

Nos mapas da Figura 1, é possível verificar o comportamento da distribuição da densidade de descargas atmosféricas no estado, considerando os períodos definidos de obtenção dos dados.
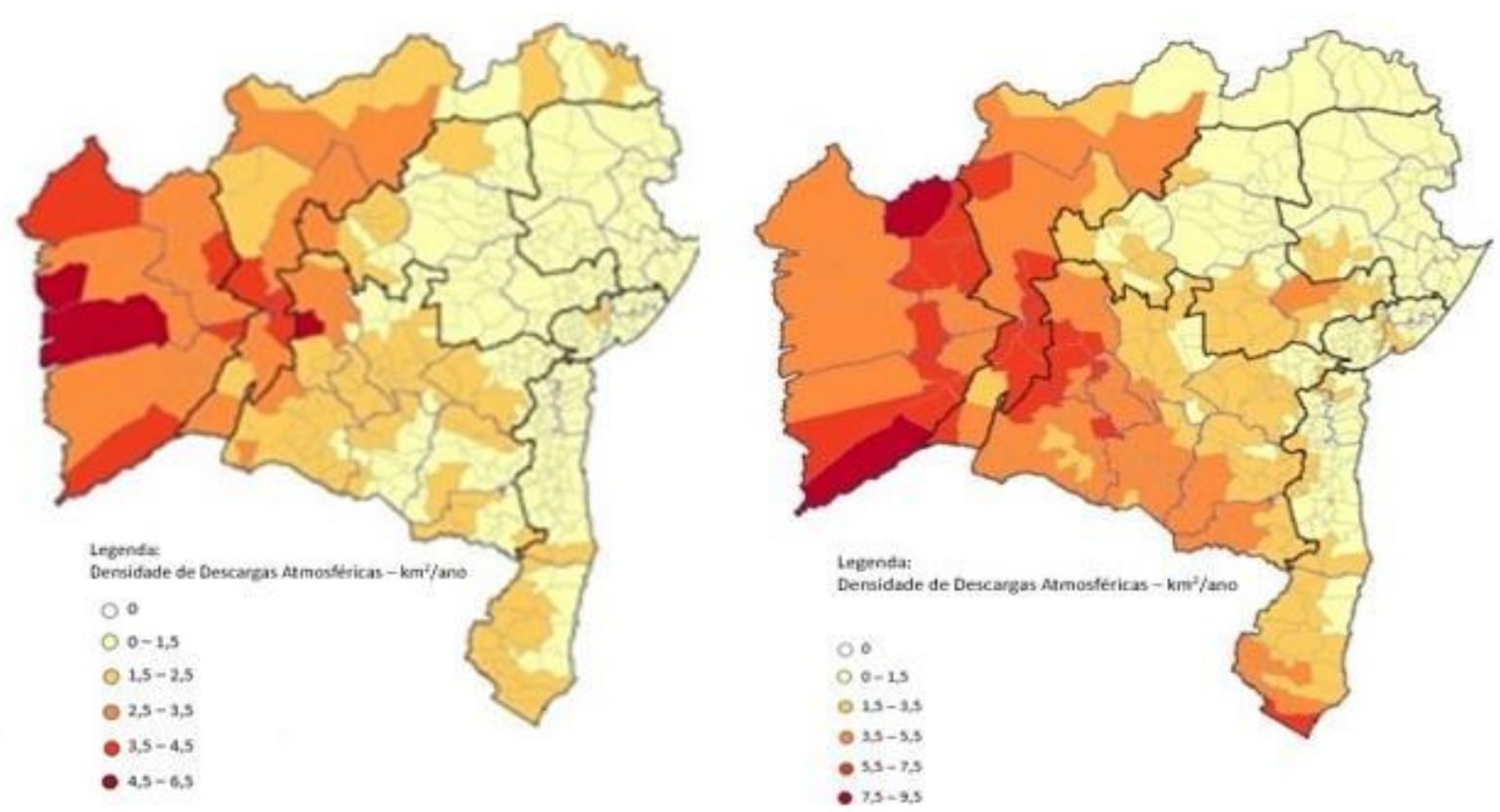

Figura 1 - Mapas da densidade de descargas atmosférica s no esta do da Bahia nos períodos de 1998 -2011 e 2016-2019.

A análise dos mapas, para ambos os intervalos, demonstram uma distribuição maior de densidades de descargas na região Oeste com um aumento da incidência na região do Centro-Sul no intervalo entre 2016 - 2019, quando comparada às médias obtidas no período entre 1998-2011. Os resultados corroboram com o contraste pontuado pelo Doutor Osmar Pinto Júnior (Bahia, 2020), expondo o fato da Bahia possuir a maior incidência de raios nas regiões Oeste e Sul. No caso, com as principais incidências na região Oeste. Sendo que os cinco primeiros municípios com maior incidência na média do intervalo entre 1998 e 2011 estão nesta região. Na Tabela 2 é possível visualizar a relação destes municípios com os seus valores de densidade.

Tabela 2 - Relação dos 05 primeiros municípios com maior densidade de raios 1998 - 2011.

\begin{tabular}{cc}
\hline Município & Densidade $\left(\mathrm{km}^{2} / \mathrm{ano}\right)$ \\
\hline Luís Eduardo Magalhães & 9,05 \\
São Desidério & 8,18 \\
Boquira & 7,79 \\
Wanderley & 7,00 \\
Muquém do São Francisco & 6,86 \\
\hline
\end{tabular}

Fonte: Elaborado pelo Autor,2020 
Além disso, uma consulta ao mapa de distribuição de descargas do Brasil no portal do ELAT para o biênio 2018 - 2019, Figura 2, demonstra a tendência de concentração da incidência de raios na região Oeste da Bahia.
Contudo, em relação, principalmente, a região Sul, Sudeste e Centro Oeste do País a Bahia possui baixos índices de densidade de descargas atmosféricas.

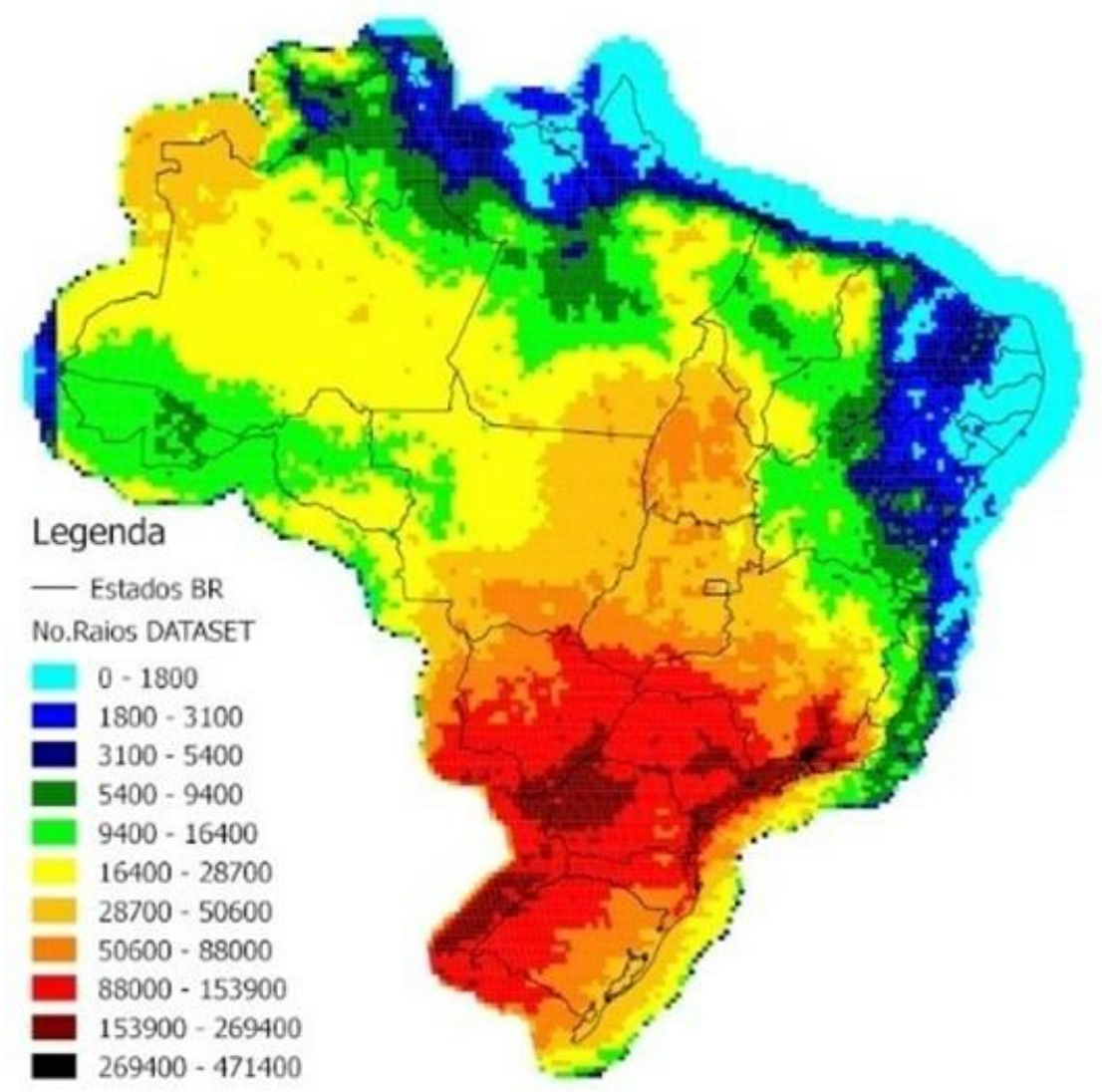

Figura 2 - Densidade de descargas atmosféricas no Brasil - biênio 2018-2019

Fonte: INPE, 2020

Já na Tabela 3, observa-se os cinco primeiros municípios com maior incidência na média de densidade de descargas atmosféricas no intervalo entre 2016 e 2019. Destaca-se que estes municípios também estão localizados na região Oeste e Centro - Sul.

Tabela 3 - Relação dos 05 primeiros municípios com maior densidade de raios 2016 - 2019.

\begin{tabular}{cc}
\hline Município & Densidade $\left(\mathrm{km}^{2} / \mathrm{ano}\right)$ \\
\hline Cocos & 6,02 \\
Santa Rita de Cassia & 4,95 \\
Jaborandi & 4,57 \\
Buritirama & 4,54 \\
Paratinga & 4,46 \\
\hline
\end{tabular}

Fonte: Elaborado pelo Autor,2020

Da análise das Tabelas 2 e 3, verifica-se uma significativa redução nos valores referentes a densidade de descargas atmosféricas da média obtida no intervalo de 1998 - 2011 em comparação com a obtida no intervalo entre 2016-2019.

Fácil observar, que o primeiro no ranking do segundo intervalo possui valor inferior ao último colocado do primeiro intervalo.
No gráfico da Figura 3, é possível se verificar uma redução na média de densidade de descargas atmosféricas, principalmente, dos municípios de Luís Eduardo Magalhães, São Desidério, Muquém do São Francisco e Boquira enquanto municípios como Cocos e Santa Rita de Cássia não apresentaram significativa variação na média de densidade de descargas nos últimos 20 anos. 


\section{Comparativo dos municípios com maior incidência entre os} intervalos

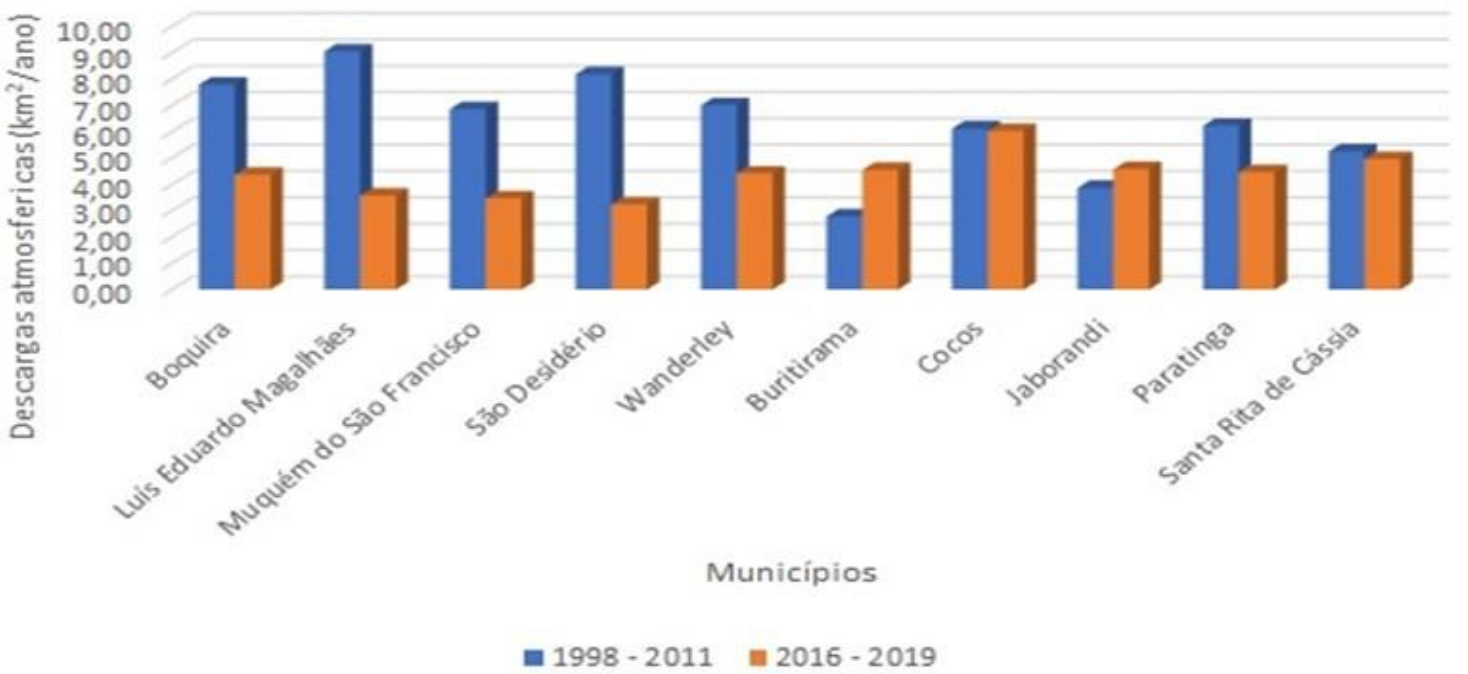

Figura 3 - Comparativo dos municípios com maior incidência de descargas entre os intervalos

Fonte: Autor, 2020.

Vale ressaltar que os resultados apresentados são importantes para projetistas de Sistemas de Proteção Contra Descargas Atmosféricas (SPDA), visto que os valores de densidade de descargas atmosféricas do município onde o projeto será implementado, devem ser observados na avaliação dos riscos, em virtude da NBR 5419:2015 assim o exigir. Desta forma, projetos de SPDA realizados nos municípios que compõem a região Oeste e Centro-Sul da Bahia, requerem níveis de risco e, consequentemente, de segurança maiores, devendo tal fato ser considerado pelos projetistas. Além disso, para Almeida (2020) e Ribeiro (2018) um conhecimento detalhado da variabilidade espacial e temporal desses eventos pode ajudar tanto no planejamento de sistemas de proteção de linhas de transmissão de energia elétrica, sistemas de telecomunicações, apoio às populações que ali habitam, bem como evitar ou mitigar danos materiais e a vidas.

Como pontuado pelo Dr. Osmar (Bahia, 2020), uma maior densidade de descargas na região Oeste, então, pode estar ligada aos fenômenos causados em algumas épocas do ano por conta do calor, visto que as nuvens ficam mais carregadas e as descargas elétricas aumentam. Como destacado nas pesquisas de Reis (2008), a região Oeste da Bahia possui aspectos físicos peculiares, que se distinguem das demais regiões do estado. As características naturais marcantes de relevo e rede hidrográfica além do clima Tropical Continental predominante, caracterizado por dois períodos bem distintos: um chuvoso e outro seco, verão e inverno, respectivamente, pode ter como resultado uma maior incidência das descargas atmosféricas na região.

Comportamento do número de mortescausadas por raios

Por fim, a distribuição do número de mortes causadas por raios no estado também foi visualizada na ferramenta VYR. Na Figura 4, observa-se a distribuição destas fatalidades por município.

Através do mapa da Figura 4, é possível verificar que a distribuição de mortes cau sadas por raios apresenta uma distribuição homogênea entre as regiões do estado, não sendo possível identificar visualmente alguma relação com os valores de densidade de descargas atmosféricas dos municípios. 


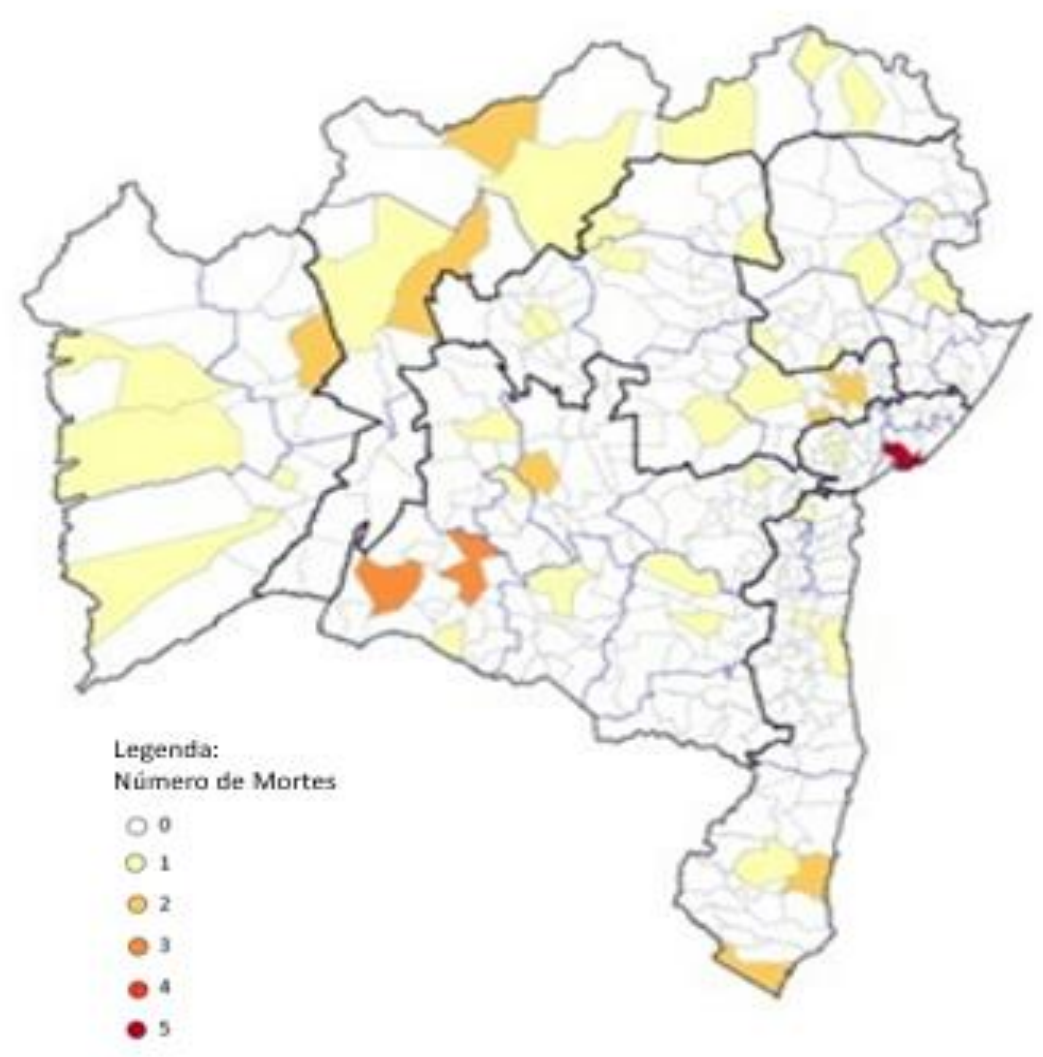

Figura 4 - Distribuição das mortes causadas por raio na Bahia

Fonte: Autor, 2020.

Entretanto, uma análise destes números a cada ano, pode fortalecer a ideia concluída por Bartolin (2019), no seu trabalho, é destacada pelo professor Alberto Brum, que a falta de informação e imprudência das pessoas é a causa de um maior número de mortes por raios (Souza, 2009). Segundo o professor, a frente fria que cobre algumas regiões aumenta a possibilidade de formação de relâmpagos, mas não justifica o alto número de atingidos (Souza, 2009). Desta forma, é provável que as mortes por raios não estejam ligadas ao nível de densidade de descargas atmosféricas na região ou outrosfatores ambientais e sim ao grau de instrução da população. Corrobora com esta conclusão os resultados obtidos por Leal (2019), que numa análise de estudo de caso demonstrou que na região Amazônica o estado do Acre, apesar de possuir uma baixa densidade de descargas, registrou quatro mortes em um único evento. As vítimas estavam transitando em um barco durante uma tempestade. Além disso, Holle (2019) e Ferreira (2018) verificaramque as grandes quantidades de mortes causadas por raios na África estavam relacionadas a falta de proteção em edificações, baixo nível socioeconômico, baixa escolaridade e falta de informação à população sobre os riscos dos raios.

Na Figura 5, visualiza-se uma redução no número de mortes causadas por raios no estado entre os anos 2000 e 2019. Tal redução pode ser devida ao aumento do grau de instrução da população, o que possibilita evitar comportamentos de risco. Em seus estudos, Couto (2017) constatou um acesso maior à educação da população baiana no período entre 2005 e 2015 . Neste período, o Índice de Desenvolvimento da Educação Básica (IDEB) cresceu de 2,6 para 3,4, mostrando uma evolução nos níveis de instrução da população. 


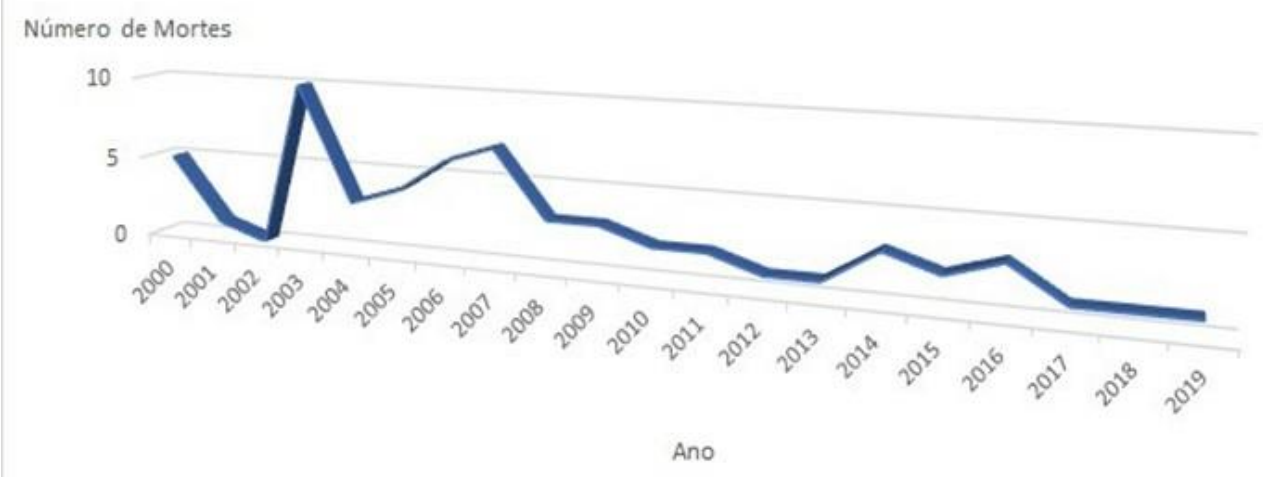

Figura 5 - Gráfico do número de mortes causadas por raio na Bahia no período de 2000 a 2019.

Fonte: Autor, 2020.

O gráfico ainda apresenta um pico de mortes ocorridas no ano de 2003, com 10 mortes, seguindo-se o ano de 2007 com sete mortes. O estado possui no período de 2000 - 2019 um total de 59 mortes. O recordista de mortes é o município de Salvador com cinco mortes, seguindo-se os municípios de Caetité e Palmas de Monte Alto com três mortes cada. Para fins de comparação, a pesquisa realizada por Leal (2019) no mesmo período entre 2009-2019 contabilizou um total de 119 mortes por raios para região da Amazónia Legal que envolve nove estados brasileiros. Segundo relatório do INPE, divulgado em 2019, o estado de São Paulo lidera o ranking brasileiro com 327 casos registrados entre 2000 e 2019 (Watanabe, 2020).

Os números demonstram que, em linhas gerais, as mortes não possuem relação com os níveis de densidade de descargas atmosféricas nos municípios, visto que o município com maior número de mortes, Salvador, possui densidade de descargas de $1,38 \mathrm{~km}^{2} /$ ano (2016-2019) ocupando a $188^{\circ}$ posição no ranking do estado.
Em seus estudos na Índia, Yadava (2020) observou que a maior densidade de descargas foi observada nas regiões montanhosas do país, mas com menos vítimas por conta da baixa densidade demográfica. Neste caso, a maior letalidade em Salvador pode estar relacionada ao fato do município possuir a maior taxa de densidade demográfica da Bahia possuindo 3859,35 hab/ $\mathrm{km}^{2}$, conforme o censo brasileiro de 2010.

Para a verificação do coeficiente de letalidade, foram considerados os dados referentes aos municípios com valores superiores a uma morte no estado no período de 2000 a 2019 . Neste caso, foram listados os municípios que obtiveram duas ou mais mortes neste período.

$\mathrm{Na}$ Tabela 4, são descritos os municípios que tiveram pelo menos duas mortes causadas por raios, seus respectivos valores médios dedensidade de descargas atmosféricas entre os dois períodos estudados (1998-2011 e 2016-2019) e a população estimada, conforme dados disponibilizados pelo IBGE. 
Tabela 04 - Municipios com mais de duas mortes com sua densidade de descargas e populaçăo estimada.

\begin{tabular}{lccc}
\hline \multicolumn{1}{c}{ Município } & $\begin{array}{c}\text { População estimada } \\
\text { - pessoas [2019] }\end{array}$ & $\begin{array}{c}\text { Média de Densidade de } \\
\text { descargas } \\
\text { (km } \mathbf{2} / \mathbf{a n o} \text { ) entre os } \\
\text { periodos }\end{array}$ & $\begin{array}{c}\text { Mortes por Raio } \\
\text { (2000-2019) }\end{array}$ \\
\hline Salvador & 2.886 .698 & 1,200 & 5 \\
Caetité & 51.081 & 2,688 & 3 \\
Palmas de Monte Alto & 21.796 & 2,858 & 3 \\
Campo Formoso & 71487 & 1,515 & 2 \\
Feira de Santana & 619.609 & 1,509 & 2 \\
Piatã & 16.984 & 2,182 & 2 \\
Prado & 28.194 & 1,536 & 2 \\
Remanso & 41.170 & 2,137 & 2 \\
Santo Estêvăo & 53.269 & 1,083 & 2 \\
Wanderley & 12180 & 5,705 & 2 \\
Xique-Xique & 46.523 & 3,851 & 2 \\
\hline
\end{tabular}

Fonte: Autor, 2020.

A partir da Tabela 4, primeiramente, foi calculado o coeficiente de letalidade com relação a densidade de descargas atmosféricas com resultados apresentados no gráfico da Figura 6.

Os resultados demonstram que o município de Salvador apresenta o maior coeficiente de letalidade quando comparado aos demais municípios. Isto ocorre, provavelmente, de vido ao município possuir um maior número de mortes causadas por raios noestado e um valor de média de densidade de descargas baixo, também, quando comparados aos demais municípios. Situação semelhante para os municípios de Santo Estevão, Feira de Santa e Campo Formoso que apresentaram os maiores coeficientes depois de Salvador. Estes municípios, apesar de apresentarem duas mortes cada, possuem baixos valores médios de densidade de descargas atmosféricas.

Já os municípios de Palma de Monte Alto e Campo Formoso possuem maior número de mortes que os municípios de Santo Estevão, Feira de Santanae Campo Formoso, mas menor coeficiente por possuírem maior número médio de densidade de descargas.

Segundo Conceição (2013), os resultados acima se devem principalmente pelas variantes área e número de raios, que definem a densidade, principal fator diferencial neste caso.

Visualizando-se o mapa de distribuição da Figura 4, o município de Salvador, Santo Estevão e Feira de Santana possuem menor área com baixa quantidade de descargas o que eleva o coeficiente quando comparado ao número de mortes.

Para os dados da Tabela 3, também foi calculado o coef iciente de letalidade com relação ao número estimado da população para cada município.

Os resultados são os apresentados pelo gráfico da Figura 7. Segundo o resultado apresentado, o município de Wanderley apresenta o maior coeficiente quando comparado aos demais municípios. Este fato ocorre porque este município apresentou duas vítimas deste fenômeno atmosférico e possui uma baixa concentração populacional. Neste sentido, o município de Wanderley apresenta a maior probabilidade de uma pessoa ser vitimada por um raio. Tudo indica, que isto se deve ao fato do referido município possuir uma alta densidade de descargas atmosféricas (a maior entre os destacados na Tabela 3 ) e uma população estimada de um pouco mais de 12.000 habitantes.

Na sequência, merece destaque os municípios de Palmas de Monte Alto e Piatã, que possuem três e duas letalidades, respectivamente, no período, é reduzido número de habitantes com relação aos demais municípios, o que os colocam como o segundo e terceiro municípios com maior possibilidade de ocorrerem letalidades causadas por raios. O município de Salvador, que possui maior número de mortes, apresentou menor coeficiente com relação ao número de habitantes na comparação entre os municípios. Isto acontece porque o município possui a maior estimativa populacional do estado. $\mathrm{O}$ mesmo fato ocorre com o município de Feira de Santana que apresentou duas mortes, mas possui a segunda maior população do estado e o segundo menor coeficiente de letalidade, quando comparados aos municípios analisados. 
Coeficiente de Letalidade em relação a média da densidade de descargas

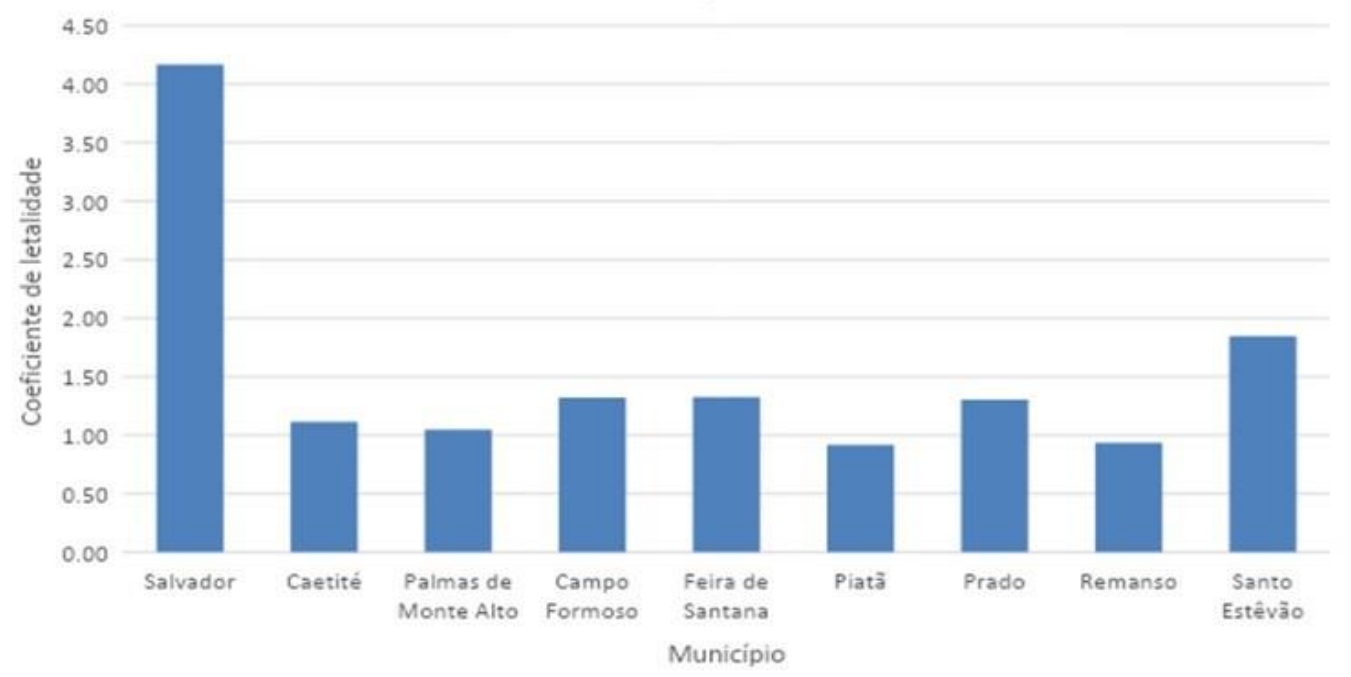

Figura 6 - Gráfico do índice de letalidade com relação a média da densidade de descargas Fonte: Autor, 2020.

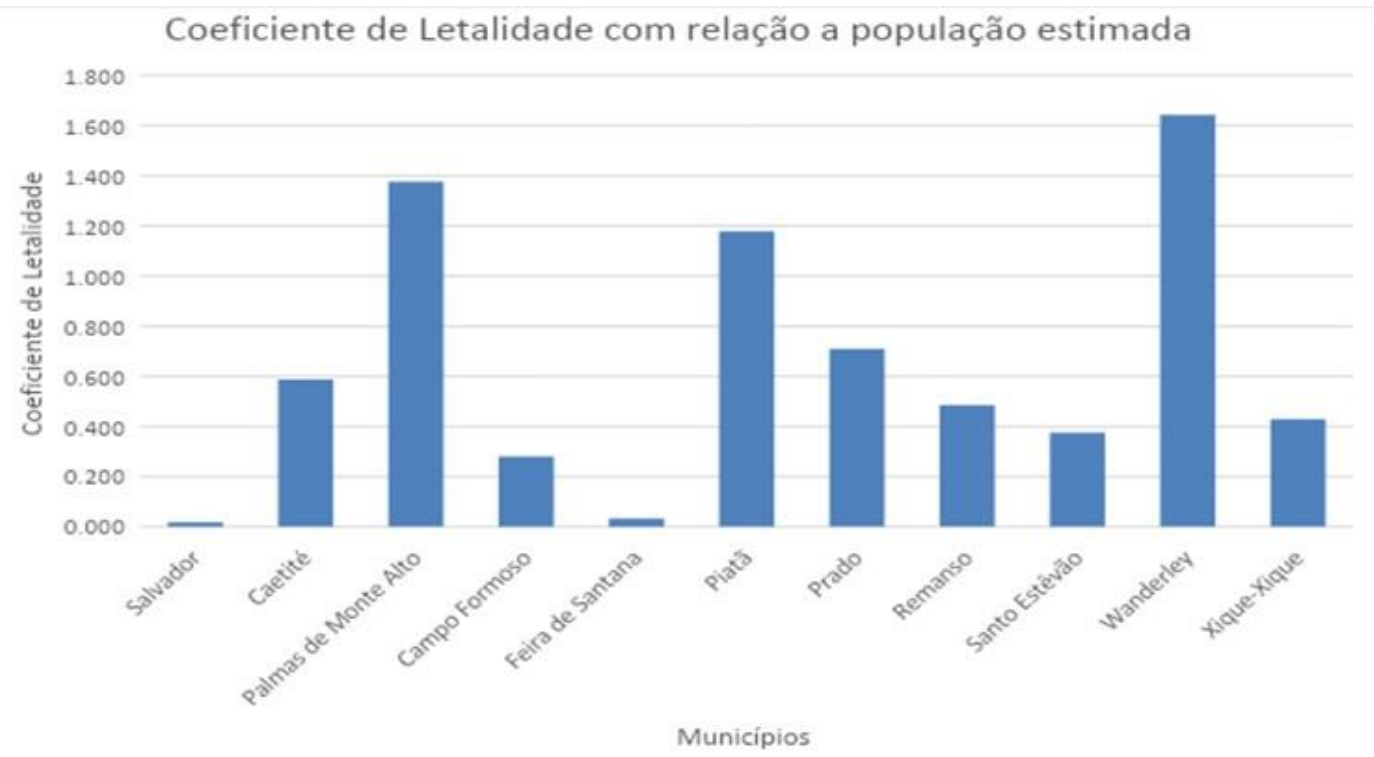

Figura 7 - Gráfico do índice de letalidade com relação a população estimada para cada 10.000 habitantes

Fonte: Autor, 2020.

\section{Conclusão}

Através da utilização de umaferramenta de análise visual, o trabalho teve como objetivo realizar um estudo sobre o comportamento da densidade de descargas atmosféricas, por município, no estado da Bahia a partir dos dados obtidos dos resultados das medições realizadas pelo INPE, bem como o comportamento das mortes por raio no estado.
Para análise, também foram utilizados os dados referentes à população estimada do estado divulgados pelo IBGE.

Numa análise visual, foi possível se identificar um comportamento de maior densidade de descargas na região Oeste da Bahia com certo aumento na região Centro-Sul quando comparadas as médias do período de 1998-2011 com as médias do intervalo de 2016-2019. Entretanto, de forma geral, foi identificada uma reduçãona densidade de 
descargas atmosféricas do período de 1998-2011 quando comparado ao período de 2016-2019.

Quanto ao número de mortes, foi visualizado um comportamento homogêneo sem identificar possível relação entre estas e a distribuição das densidades de descargas atmosféricas.

Comparando-se os números no período entre os anos 2000 e 2019, observou-se uma redução no número de mortes causadas por raios. Tal fato pode estar relacionado ao aumento do nível de instrução da população.

Através dos cálculos de coeficientes de letalidade foram obtidos resultados que demonstraram que, com relação a densidade de descargas atmosféricas, o município de Salvador possui a maior possibilidade de letalidade. Entretanto, tomando-se como base a relação de fatalidades com a estimativa populacional, o município de Wanderley possui a maior probabilidade de morte causada por raios.

Como trabalho futuro sugere-se a análise da influência de outros fatores no comportamento da densidade de descargas atmosféricas e número de mortes causadas por raios como clima, urbanização, relevo e densidade demográfica para o estado da Bahia.

\section{Agradecimentos}

Os autores agradecem ao Instituto Federal de Educação, Ciência e Tecnologia da Bahia IFBA pelo apoio dado ao estudo.

\section{Referências}

Alconforado, F. A.G, 2003. Os Condicionantes do Desenvolvimento do Estado da Bahia. Tese de Doutorado em Ciências Humanas Universidade de Barcelona, 2003. Departamento de Geografia Física e Análise Geográfica Regional.

Albrecht, I.R., Almeida A.P., Calheiros P.J.A., Machado,L.A.T., Santos, R.D.C., Neto, H.B.L., Souza, C.F. 2020 A Distribuição Espacial das Descargas Elétricas na Região Central da Bacia Amazônica e o uso da Árvore de Decisão em dados de radar meteorológico para sua Classificação. Anais do XI Workshop de Computação Aplicada à Gestão do Meio Ambiente e Recursos Naturais. SBC OPEN LIB.

Almeida, C.A, Souza, S.R.J, Pompeu, M. M., Ribeiro, N.M.W., 2020. Variabilidade Espacial e Temporal de Descargas Atmosféricas na Região Norte. Revista Brasileira de Geografia Física v.13, n.05 (2020) 2074-2085.
Antonescu B, Cárbunaru F, 2018. LightningRelated Fatalities in Romania from 1999 to 2015. Weather, Climate and Society. American Meteorological Society. 10.

Bahia, T. 2020. Bahia registra mais de 100 mil raios em 24horas. Disponível em: https://g1.globo.com/ba/bahia/noticia/2020/10/ 28 bahia-registra-mais-de-100-mil-raios-em24-horas-e-fazenda-e-incendiada-pelas-

descargas-eletricas. ghtml. Acesso: 30 jan. 2020.

Banwari, M, 2021. Tendência de 20 anos (19962015) em mortes relacionadas a raios na subregião de Transkei da África do Sul. Indian Journal of Health Research \& Development. 9 Edição 12.

Bortolin, E., Galvani, E., 2017. Atmospheric discharges and the geographic aspecto: case study of the ocurrences in the hydrographic basin of the Paraíba do Sul (São Paulo) river and implications in the society. Revista Entre Lugar. 8, 227-247.

Conceição, R. P, André, I. R. N, Azevedo, T.S, 2013. Espacialização das Descargas Elétricas Atmosféricas no Estado de São Paulo para os anos de 2005-2006, e suas Consequências. Revista Brasileira de Climatologia. Ano 9. 13, no.0.

Cooper, M.A., Ab Kadir, M.Z.A., Syakura, A.R., Elistina, A.B, 2018. Comparative Study on Lightning Fatality Rate in Malaysia between 2008 and 2017. 34th International Conference on Lightning Protection. ICLP 2018.

Couto, M.F., Ribeiro, L.C.S., 2017. Estrutura produtiva e nível de escolaridade na Bahia. Laboratório de Análises Territoriais e Setoriais - LATES. Texto para Discussão No 01-2017.

DATASUS. Ministério da Saúde, 2020. Sistema de Informações sobre Mortalidade. Disponível: http://tabnet.datasus.gov.br/cgi/deftohtm.exe?si m/cnv/obt10uf. def. Acesso: 30 jan. 2020.

Deiro, H., Novais, R., 2021. Visualize Your Region-VYR. Disponível em: http://vyr.hugodeiro.com. Acesso: 30 de abril 2021.

ELAT. Grupo de Eletricidade Atmosférica Instituto Nacional de Pesquisas Espaciais, 2020. Cartilha de proteção contra raios. Disponível :http://www.inpe.br/webelat/homepage/menu/p rotecao/cartilha.de.protecao.contra.raios.php. Acesso: 30 jan. 2020.

Eler, G., 2020. Brasil o País que, mais caem Raios no Mundo. Revista Superinteressante. Disponível:

https://super.abril.com.br/especiais/brasil-opais-dos-raios. Acesso: 30 jan. 2020. 
Fan, X., Yin, Q., Zhang, Y., Zhuang, Y., Wang, F., Du, H., Huang, X., Chen, S., Chen. L. 2021. Lightning Fatalities in China, 2009-2018. Journal of Agricultural Meteorology 77(2): 150159.

Ferreira, C.F., Santos, R.M.A., Silva, S.S.R., Carvalho, R.A.N., Pereira, L.S.P., Andrade, A.H.J., 2018. Mortalidade por Raio Atmosférico. Brazilian Journal of Surgery and Clinical Research - BJSCR 21, 106-110 (Dez 2017 - Fev 2018) .

Garcia, R, 2013. Poluição e urbanização fazem incidência de raios aumentar em São Paulo. Disponível

:https://m.folha.uol.com.br/ciencia/2013/09/ 1338941-poluicao-e-urbanizacao-fazemincidencia-de-raios-aumentar-em-sao-paulo. shtml. Acesso: 30 jan. 2020.

Gin, R. B. B., Filho, A.J.P., Beneti, C.A.A., Guedes, R.L, 2012. Estudo das descargas elétricas atmosféricas no sul e sudeste do brasil: Análise preliminar. XI Congresso. Brasileiro de. Meteorologia.

Gomes, D. A. L. L. R, 2019. Estudo da incidência de descargas atmosféricas na região de governador Valadares. Seminário de Iniciação Científica.

Grégio, A. R. A. et al, 2009. Técnicas de Visualização de Dados aplicadas à Segurança da Informação.

Disponível :http://www.lac.inpe.br/ rafael.santos/Docs/SB SEG/ 2009/sbseg2009.pdf. Acesso: 30 jan. 2020

Heilmann, A, Fernandes, C., Leite, E.A, Pinheiro, L.C, 2018. Características das tempestades com raios no litoral do Paraná. Revista Brasileira de Climatologia. 22, no.0

Holle, L.R., Cooper, A.M., Tushemereirwe, R., 2019. Mitigating the Hazard of Lightning Injury and Death across Africa. IntechOpen.

INPE. Instituto Nacional de Pesquisas Espaciais, 2007. ELAT faz primeira avaliação detalhada dos prejuízos causados por raios no país. 2007. Disponível:

http://www.cea.inpe.br/webelat/homepage/men u/noticias/

informativo/index.php?informativo $=38$ > . Acesso: 30 jan. 2020

INPE. Instituto Nacional de Pesquisas Espaciais, 2011. Divulgado novo ranking de incidência de raios.

Disponível: http://www.inpe.br/noticias/noticia.php?Cod Noticia $=2518>$. Acesso: 30 jan. 2020

INPE. Instituto Nacional de Pesquisas Espaciais, 2013. Ranking 1998 -2011 LIS mortes 2000 2011 alfa. Disponível :http://
www.inpe.br/webelat/docs/Densidade_de_Raio s_por_Municipio_2013_03_28.pdf>. Acesso: 30 jan. 2020

INPE. Instituto Nacional de Pesquisas Espaciais, 2020. Mapa do Biênio 2018/2019. Disponível: http://www.inpe.br/webelat/homepage/menu/in for/incidencia.de.descargas.no.pais.php>

Acesso: 15 fev. 2020.

Lau, H. K., Kaplan, J.O, 2021. The WGLC Global Gridded Lightning Climatology and Timeseries. Earth System Science Data.

Leal, A., Ferreira, E.R., Matos, N.L.W., Gomes, N.M., 2019. Lightning deaths and injuries in the razilian Amazon Region in the period of 20092019. 2019 International Symposium on Lightning Protection (XV SIPDA), São Paulo, Brazil, 30th September - 4th October 2019.

Lyu, W., Xu, L., Zheng, D., Zhang, Y., Zhang, W., 2018. Lighting climatology over the northwest Pacific region: An 11-year study using data from the World Wide Lightning Location Network. Atmospheric Research 210, 41-57.

Paulino, J. O. S, 2016. Proteção de Equipamentos Elétricos e Eletrónicos contra Surtos Elétricos em instalações. Tese (Doutorado), Lagoa Santa, 2016.

Pompeu, M. M., Ribeiro, N.M.W., Queiroz, B.C.J., Almeida, C.A., Souza, S.R.J., Silva, S.C.C., 2018. Previsão de raios de índices termodinâmicos da atmosfera, na região de Belém do Pará. Revista Brasileira de Geografia Física 11, 1186-1201.

Porras, L., Younes, C., Herrera, J., 2018. Cloud-toground lightning activity in Colombia: A 14year study using lightning location system data. Atmospheric Research 203, 164-174.

Reis, F. R. dos, 2008. Analise da Criação do estado do Rio São Francisco sob a Caracterização Socioeconômica da Região Oeste do Estado da Bahia. 2008. Disponível: http://observatoriogeograficoamericalatina.org. mx/egal12/Geografiasocioeconomica/

Geografiadelapoblacion/33.pdf>. Acesso: 30 jan. 2020.

Ribeiro, N.M.W., Silva, S.C.C, Almeida, C.A., Queiroz, B.C.J., Souza, S.R.J., Pompeu, M.M, 2018.Lightning characteristics associated to severe storm cases, which impacted the cities of Belem and Santarem, in Amazonia. Revista Brasileira de Geografia Física v11, 1202-1214.

Romero, F., 2011.Avaliação dos comportamentos dos Campos eletromagnéticos gerados por descargas atmosféricas nuvem - terra. Tese (Doutorado) - Universidade de São Paulo, São Paulo, USP, 2007. Disponível :https://teses.usp.br/teses/disponiveis/86/86131 


\section{I}

tde-26032007-

162159/publico/FabioRomero_Dissertacao_de _mestrado.pdf. Acesso em: 3 jul. 2011.

Santos, A.P.P, Júnior, O.P, Souza, E.B, Azambuja, R, Santos, S.R.Q, 2016. Variabilidade espaçotemporal e identificação de eventos extremos de descargas atmosféricas no estado de São Paulo durante o verão. Revista Brasileirade Geografia Física 9, 346-352.

Santos, A.P.P, Júnior, O.P, Souza, E.B, Azambuja, R, Santos, S.R.Q, 2016. Eventos climáticos extremos de descargas atmosféricas sobre o estado de São Paulo. Parte I: Aspectos Anuais e Sanzonais. Revista Brasileira de Geografia Física 9, 1346-1356.

Seltzer, M., Alber, R., Sugier, J., Enno, S., 2020. Lightning flash density in Europe based on 10 years of ATDnet data. Atmospheric Research 235 (2020) 104769. Relação Entre o Total de Raios e os Raios Nuvem-Solo Sobre o Leste da Amazônia. Revista Brasileira de Geografia Física 13, 782-797.

Sodré, C.R.C., Ferreira, S.B.D., Ventura, O.J., Costa, W. P. C., Everaldo, B.S., Moraes, C.B., 2020.

Sousa, D. P, Filho, O. J. L, Philadelpho, P.P.N, Lima, A.L.D.S, 2012. Verificação da relação entre urbanização e incidência de raios nos municípios do Brasil. Cadernos do IME - Série Estatística. 32, 33-48.

Souza, A. R. J, 2009. Raios já mataram quatro no estado em 2009. Disponível :https://atarde.uol.com.br/bahia/salvador/notici as/ 1279536-raios-ja-mataram-quatro-noestado-em-2009. Acesso: 30 jan. 2020.

Tian, W., Qie, X,. Qie, K., 2021. Increasing trend of lightning activity in the South Asia region. Science Bulletin 66, 78-84.

Tufte, E. R., 2001. The Visual Display of Quantitative Information. Graphics Pr; $2^{\mathrm{a}}$ edição.

Watanabe,F., 2020. Raios em São Paulo diminuem com isolamento social e menos poluição.2020. Disponível:

https://www1.folha.uol.com.br/ambiente/2020/ 06/raios-em-sao-paulo-diminuem-comisolamento-social-e-menospoluicao.shtml\#: :text=S\%C3\%A3o\%20Paulo $\% 20 \% \mathrm{C} 3 \% \mathrm{~A} 9 \% 20 \mathrm{o} \% 20$ estado,segundo\%20ou tro\%20estudo\%20do\%20Elat. Acesso: 15 maio 2020

Yadava, K.P., Soni, M., Verma, S., Kumar, A.S., Payra, S., 2020. The major lightning regions and associated casualties over India. Natural Hazards 101, 217-229 